\title{
Constructive Interference Precoding for Reliable Non-Orthogonal IoT Signaling
}

\author{
Tongyang $\mathrm{Xu}^{*}$, Fan Liu*, Ang $\mathrm{Li}^{\dagger}$, Christos Masouros* and Izzat Darwazeh* \\ * Department of Electronic and Electrical Engineering, University College London, London, UK \\ ${ }^{\dagger}$ School of Electrical and Information Engineering, University of Sydney, Australia \\ Email: *\{tongyang.xu.11, fan.liu, c.masouros, i.darwazeh\}@ucl.ac.uk, †ang.li2@sydney.edu.au
}

\begin{abstract}
Efficient signal waveform design is paving the way to next generation Internet of Things (NG-IoT) communications. Non-orthogonal spectrally efficient frequency division multiplexing (SEFDM) waveform brings bandwidth saving advantages but at the cost of complex signal processing in IoT devices. Power efficiency is the main concern in IoT applications since IoT devices have limited battery life and cannot easily recharge after deployment. Therefore, signal processing in each IoT device should be as simple as possible. Precoding is applied as the strategy at transmitters to pre-cancel the self-created inter carrier interference (ICI) caused by the non-orthogonal sub-carrier packing, and to facilitate low-complexity IoT receivers. However, the typical zero forcing (ZF) precoding results in performance degradation due to the ill-conditioned precoding matrix. To achieve reliable communications using SEFDM signals, a robust constructive interference (CI) precoding method is employed, which constructively converts interference power to useful signal power. Thus, signal-to-noise ratio (SNR) is effectively improved leading to better performance than $\mathrm{ZF}$ precoding. This work initially studies the performance of precoding methods in simulation. Furthermore, the CI precoding is practically tested overthe-air on a multi-user multiple input multiple output (MIMO)SEFDM software defined radio NG-IoT platform.
\end{abstract}

Index Terms-Precoding, IoT, non-orthogonal, software defined radio, prototyping, USRP, constructive interference, MIMO, SEFDM, spectral efficiency.

\section{INTRODUCTION}

Internet of Things (IoT) is evolving into the next stage, which would employ new signal waveforms to extend signal coverage, boost power efficiency and improve data rate in low power wide area network (LPWAN) communications scenarios. Non-orthogonal signal waveforms would be the direction due to the high spectral efficiency. The non-orthogonal waveform design includes candidates such as filterbank based multicarrier (FBMC) [1], generalized frequency division multiplexing (GFDM) [2] and universal-filtered multi-carrier (UFMC) [3]. However, the complex pulse-shaping-based processing makes them unattractive for IoT applications. IoT devices would consume extra power to generate and recover those signals. One potential waveform candidate is the bandwidth saving multicarrier signal waveform spectrally efficient frequency division multiplexing (SEFDM) [4], which has been successfully implemented in wireless $60 \mathrm{GHz}$ mm-wave [5] and visible light communication (VLC) [6]. The benefit of this waveform is bandwidth saving and straightforward signal generation. However, signal detection is challenging to recover signals from non-orthogonal sub-carrier packing inter carrier interference (ICI) [5]. Thus, SEFDM signals are normally used in uplink channels, in which all the signal processing is operated at base station.

In downlink channels, to avoid extra power consumption of signal processing in IoT devices, precoding is preferred at the transmitter. The purpose of precoding is to modify signal characteristics before transmission. In this way, subcarrier interference is automatically cancelled at the receiver. The widely used precoding strategy is zero forcing (ZF) [7], which is low-complexity and has been tested in precoded SEFDM [8]. However, the ZF precoding will enhance noise effect and therefore greatly affect performance due to the illconditioned precoding matrix. A recently proposed precoding method, termed constructive interference (CI) precoding [9], [10], optimizing the precoding matrix in a symbol level, can constructively convert interference power to useful signal power. Therefore, the noise enhancement impact is mitigated.

This work studies, through simulation and experimental investigation, the adaptation of the CI precoding in dealing with non-orthogonal signal waveform interference. It first evaluates spectral efficiency improvement by using the CI precoding in simulation. Although most IoT applications are based on single-antenna single-user communication scenarios, this work extends to a more challenging multi-antenna multiuser scenario. Therefore, a real-time multi-antenna multi-user software defined radio universal software radio peripheral (USRP) [11] based next generation IoT (NG-IoT) platform is built with a realistic over-the-air transmission link. It should be noted that the platform is scalable and it can support singleantenna single-user communications as well. The CI precoding is then implemented on the platform and practical results are collected to show the interference robustness of the CI precoded SEFDM over the typical ZF precoded SEFDM.

\section{SignAl Model}

The cellular based IoT standard, narrowband IoT (NB-IoT) [12], defines a narrowband QPSK modulated orthogonal frequency division multiplexing (OFDM) signal occupying 12 sub-carriers in $180 \mathrm{kHz}$ bandwidth. Therefore, the evolution from NB-IoT to non-orthogonal NB-IoT is straightforward by replacing OFDM signals with SEFDM signals.

The waveform compression principle is illustrated in Fig. 1. The bandwidth of each sub-carrier is fixed to $15 \mathrm{kHz}$ following the NB-IoT standard [13]. The OFDM waveform, 


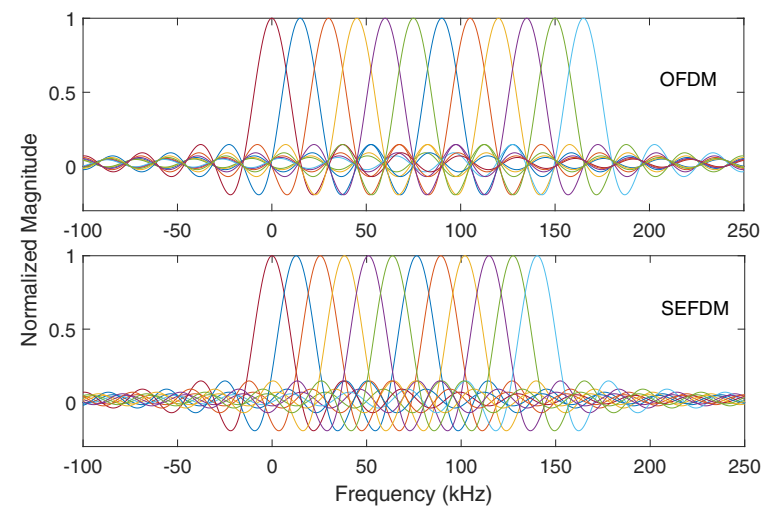

Fig. 1. Sub-carrier packing schemes for different multicarrier signals, OFDM (12 sub-carriers, bandwidth is $B)$. SEFDM (12 sub-carriers, bandwidth compression factor $\alpha=0.85$, bandwidth is $\alpha \times B$ ).

shown in Fig. 1, packs sub-carriers orthogonally at $15 \mathrm{kHz}$ spacing without ICI. However, for the SEFDM signal in Fig. 1 sub-carriers are packed closer than $15 \mathrm{kHz}$ breaking orthogonality. Thus, the total occupied signal bandwidth of SEFDM is narrower and interference is introduced.

The SEFDM signal is mathematically expressed as

$$
X_{k}=\frac{1}{\sqrt{N}} \sum_{n=0}^{N-1} s_{n} \exp \left(\frac{j 2 \pi n k \alpha}{N}\right),
$$

where $N$ is the number of sub-carriers, $s_{n}$ is the QPSK symbol modulated on the $n^{\text {th }}$ sub-carrier, $X_{k}$ is the $k^{\text {th }}$ time sample with $k=1, \ldots, N, \alpha=\Delta f \cdot T$ is the bandwidth compression factor, where $T$ is one SEFDM symbol time period and $\Delta f$ is the sub-carrier spacing. The SEFDM signal can be simply generated via inverse fast Fourier transform (IFFT) based on the techniques developed in [14]. To simplify the expression, a matrix format of the SEFDM signal is defined as

$$
X=\mathbf{F} S,
$$

where $\mathbf{F}$ is the fixed $N \times N$ sub-carrier matrix with its $(k, n)^{t h}$ entry being expressed as $e^{\frac{j 2 \pi n k \alpha}{N}}$ and $S=\left[s_{1}, \ldots, s_{N}\right]^{T}$ indicates an $N$ length vector of transmitted symbols.

To simplify the signal expression, additive white Gaussian noise (AWGN) channel is assumed. Therefore, the demodulated signal at the receiver is given as

$$
R=\mathbf{F}^{*}(X+Z)=\mathbf{F}^{*} \mathbf{F} S+\mathbf{F}^{*} Z=\mathbf{C} S+Z_{\mathbf{F}^{*}},
$$

where $\mathbf{F}^{*}$ indicates the demodulation sub-carrier matrix with elements equal $e^{\frac{-j 2 \pi n k \alpha}{N}}, Z$ denotes AWGN and $Z_{\mathbf{F}^{*}}$ is the AWGN after demodulation. The correlation matrix $\mathbf{C}$, representing the self-created ICI, is defined as

$$
\begin{aligned}
c_{m, n} & =\frac{1}{N} \sum_{k=0}^{N-1} e^{\frac{j 2 \pi m k \alpha}{N}} e^{-\frac{j 2 \pi n k \alpha}{N}} \\
& =\left\{\begin{array}{ll}
1 & , m=n \\
\frac{1-e^{j 2 \pi \alpha(m-n)}}{N\left(1-e^{\frac{j 2 \pi \alpha(m-n)}{N}}\right)} & , m \neq n
\end{array}\right\},
\end{aligned}
$$

where $m, n$ are two arbitrary sub-carrier indices. The diagonal elements indicate sub-carriers auto-correlation when $m=n$ while the non-diagonal elements indicate cross interference among sub-carriers, and are dependent only on the sub-carrier spacing $\alpha$, and not on the channel response.

\section{PRECOding}

\section{A. System Model}

The existing 3GPP NB-IoT standard [13] defines singleantenna single-user scenario. For the sake of generality, a multi-antenna multi-user system architecture, shown in Fig. 2, is considered in this work. The MIMO precoding, aiming to cancel multi-user interference, employs the typical ZF strategy [7]. The waveform precoding, which applies the CI precoding technique, is the work we are focusing.

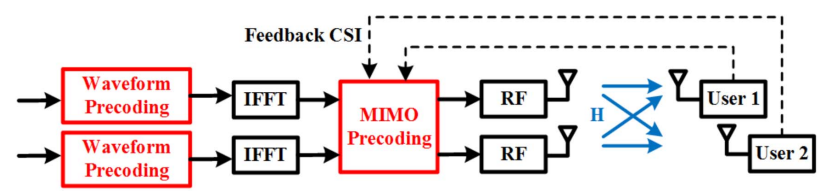

Fig. 2. Architecture of two-stage precoding multi-user MIMOSEFDM.

\section{B. MIMO Precoding}

Assume the MIMO channel is $\mathbf{H}$, the received symbol contaminated by MIMO channel and AWGN is re-defined as

$$
Y=\mathbf{H} U+Z
$$

where $U=\left[x_{11}, x_{21}, \ldots, x_{b 1}\right]$ is the signal vector coming from $b$ antennas at the first time slot. For example, $x_{11}$ is the first sample of an SEFDM signal from the first antenna and $x_{21}$ indicates the first sample of an SEFDM signal from the second antenna.

In order to remove the effect of $\mathbf{H}$, time orthogonal pilot symbols [15] are used to estimate the channel state information (CSI) and further to precode signals at the transmitter. In this case, the MIMO channel effect, $\mathbf{H}$ and waveform interference, $\mathbf{C}$ can be separated and processed in time-domain and frequency-domain, respectively. Therefore, prior to signal transmission, the signal $U$ has to go through an MIMO precoding operation as

$$
\tilde{U}=\hat{\mathbf{H}}^{H}\left(\hat{\mathbf{H}} \hat{\mathbf{H}}^{H}\right)^{-1} U
$$

Therefore, the received signal after MIMO channels and AWGN is expressed as

$$
\begin{aligned}
\hat{Y} & =\mathbf{H} \tilde{U}+Z, \\
& =\mathbf{H} \hat{\mathbf{H}}^{H}\left(\hat{\mathbf{H}} \hat{\mathbf{H}}^{H}\right)^{-1} U+Z, \\
& =\hat{U}
\end{aligned}
$$

where $\hat{\mathbf{H}}$ is the estimated channel matrix and $\hat{U}$ indicates the estimated time-domain sample, which may be affected by imperfect channel estimation and noise. 


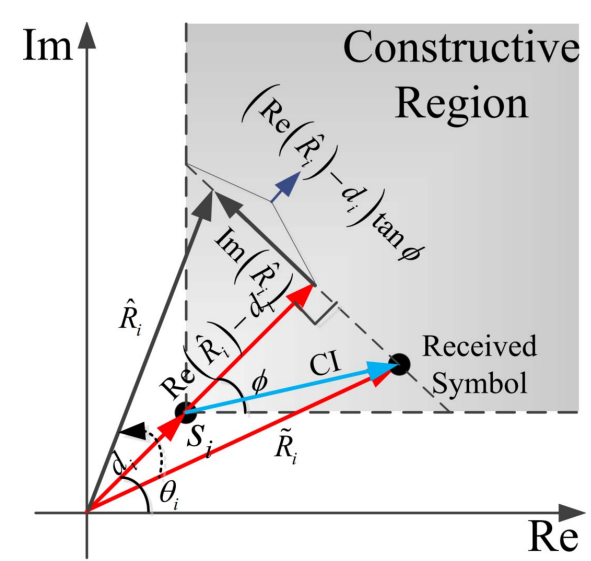

Fig. 3. The principle of constructive interference.

\section{Waveform Precoding}

The self-created ICI may contribute constructively or destructively to the power of the useful signal [9], [10]. For a given constellation point, the $\mathrm{CI}$ is defined as the interference that pushes the received symbol away from the decision thresholds, such that the probability of correct decisions can be improved [9]. This is illustrated in Fig. 3, where the grey area denotes the constructive region of a QPSK symbol, and the blue vector represents the constructive interference. Inspired by the above observation, here we aim to rotate all the ICI as the $\mathrm{CI}$, such that the received symbol may fall into the constructive region.

Assume the MIMO channel is precoded in (7) and both the transmitter and the receiver are equipped with a single antenna. Considering (3), a new equation is given as

$$
R=\mathbf{C} V+Z_{\mathbf{F}^{*}}
$$

where $V=\mathbf{W} S$ is the precoded symbol vector, $\mathbf{W}=$ $\left[\mathbf{w}_{0}, \mathbf{w}_{1}, \ldots, \mathbf{w}_{N-1}\right] \in \mathbb{C}^{N \times N}$ is the precoding matrix, and $\mathbf{w}_{i}$ is the precoding vector of the $i^{t h}$ sub-carrier. The received symbol for the $i^{t h}$ sub-carrier after demodulation can be given by

$$
R_{i}=\mathbf{c}_{i}^{T} \sum_{k=0}^{N-1} \mathbf{w}_{k} s_{k}+Z_{\mathbf{F}^{*}, i}=\mathbf{c}_{i}^{T} \sum_{k=0}^{N-1} \mathbf{w}_{k} e^{j \theta_{k}}+Z_{\mathbf{F}^{*}, i}, \forall i,
$$

where $s_{k}$ is the QPSK symbol on the $k^{t h}$ sub-carrier, $\theta_{k}$ is the phase of the $k^{t h}$ symbol, and $\mathbf{c}_{i}$ is the $i^{\text {th }}$ vector of $\mathbf{C}$.

We then investigate the constraint that forces the received symbol to fall into the constructive region. Let us consider a noise-free received symbol $\tilde{R}_{i}=\mathbf{c}_{i}^{T} \sum_{k=0}^{N-1} \mathbf{w}_{k} s_{k}$ in the CI region as shown in Fig. 3. For the ease of analysis, we rotate $\tilde{R}_{i}$ into the reference coordinate system of the desired symbol $s_{i}$, which yields

$$
\hat{R}_{i}=\tilde{R}_{i} e^{-j \theta_{i}}=\mathbf{c}_{i}^{T} \sum_{k=0}^{N-1} \mathbf{w}_{k} e^{j\left(\theta_{k}-\theta_{i}\right)} .
$$

It can be observed that after rotation, the real part of $\hat{R}_{i}$ is aligned with the direction of the symbol $s_{i}$, and will always constructively contribute to the received symbol if it is greater than zero. In order to keep $\hat{R}_{i}$ in the constructive region, the imaginary part should satisfy that

$$
\left|\operatorname{Im}\left(\hat{R}_{i}\right)\right| \leq\left(\operatorname{Re}\left(\hat{R}_{i}\right)-d_{i}\right) \tan \phi,
$$

where $\operatorname{Re}\left(\hat{R}_{i}\right)$ and $\operatorname{Im}\left(\hat{R}_{i}\right)$ represent the real and imaginary parts of $\hat{R}_{i}$, respectively, $\phi=\pi / M$ with $M$ being the modulation order of the MPSK, finally $d_{i}$ denotes the modulus of the transmitted constellation symbol. By the above analysis, the CI constraint can be given by

$$
\begin{aligned}
& \left|\operatorname{Im}\left(\mathbf{c}_{i}^{T} \sum_{k=0}^{N-1} \mathbf{w}_{k} e^{j\left(\theta_{k}-\theta_{i}\right)}\right)\right| \\
& \leq\left(\operatorname{Re}\left(\mathbf{c}_{i}^{T} \sum_{k=0}^{N-1} \mathbf{w}_{k} e^{j\left(\theta_{k}-\theta_{i}\right)}\right)-d_{i}\right) \tan \phi .
\end{aligned}
$$

It is clear from above that, the SNR of $\hat{R}_{i}$ can be maximized by maximizing $d_{i}$, which represents the minimum distance that the symbol can be pushed away from the decision thresholds. We therefore consider the following optimization problem

$$
\begin{array}{rl}
\max _{\mathbf{w}_{k}, d} & d \\
\text { s.t. } & \left|\operatorname{Im}\left(\mathbf{c}_{i}^{T} \sum_{k=0}^{N-1} \mathbf{w}_{k} e^{j\left(\theta_{k}-\theta_{i}\right)}\right)\right| \\
& \leq\left(\operatorname{Re}\left(\mathbf{c}_{i}^{T} \sum_{k=0}^{N-1} \mathbf{w}_{k} e^{j\left(\theta_{k}-\theta_{i}\right)}\right)-d\right) \tan \phi, \forall i, \\
& \left|\sum_{k=0}^{N-1} \mathbf{w}_{k} e^{j \theta_{k}}\right|^{2} \leq P,
\end{array}
$$

where $P$ is the transmit power budget. The above problem is convex in $\mathbf{w}_{k}$ and $d$, and can be optimally solved via standard approaches. To accelerate the acquisition of the solutions, we employ the low-complexity algorithm proposed in [10] for the CI precoding scheme.

\section{Simulation Evaluation}

We focus on a scenario where IoT devices are normally deployed in fixed locations so they are effectively stationary. Therefore, the channel is nearly static and no obvious frequency fading exists. The simulation is undertaken in AWGN channel for function validation, with 12 sub-carriers modulated by QPSK. Effective spectral efficiency $S E_{\text {eff }}$, which is related to bit error rate (BER), is calculated as

$$
S E_{e f f}=\frac{1}{\alpha} \times(1-B E R) \times \log _{2} M
$$

where (1-BER) indicates non-error bit rate and $M=4$ for QPSK modulation.

Systems with three different bandwidth compression factors are evaluated. The first is an SEFDM system with $\alpha=0.9$, where as shown in Fig. 4 that the ZF precoding and the CI 


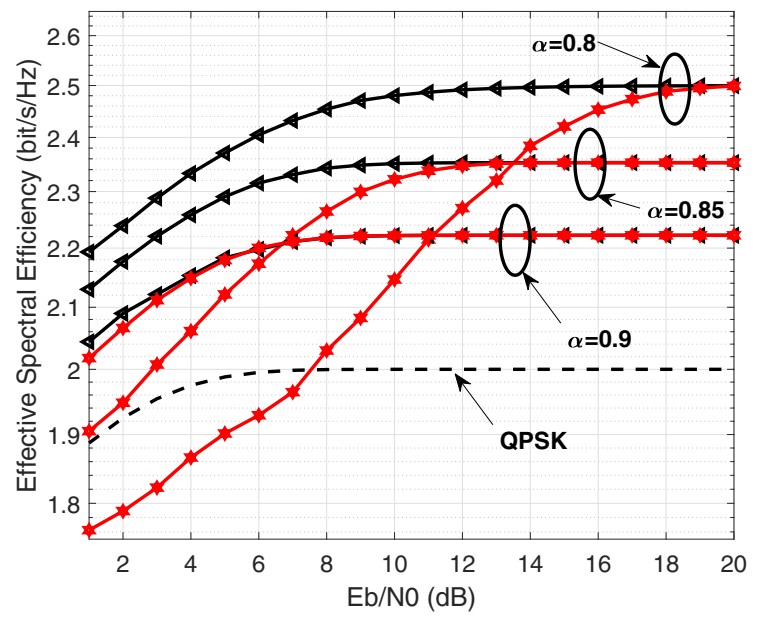

Fig. 4. Spectral efficiency comparison at different $\alpha$. Triangular curves indicate $\mathrm{CI}$ precoding and star curves indicate $\mathrm{ZF}$ precoding.

precoding performances are almost equal and both reach the maximum spectral efficiency $2.22 \mathrm{bit} / \mathrm{s} / \mathrm{Hz}$ at $\mathrm{Eb} / \mathrm{NO}=9 \mathrm{~dB}$, which is greater than the $2 \mathrm{bit} / \mathrm{s} / \mathrm{Hz}$ spectral efficiency of QPSK. In this case, there is almost no benefit for precoding given the little ICI [5] at the benefits of CI precoding.

To introduce higher interference, the bandwidth compression is increased to $15 \%$ and the effective spectral efficiency of each system is shown in Fig. 4. It is shown that CI precoding significantly outperforms $\mathrm{ZF}$ precoding up to $\mathrm{Eb} / \mathrm{N} 0=13 \mathrm{~dB}$. As is shown in Fig. 5, with the increase of bandwidth compression, systems are more likely to have small eigenvalues. The small eigenvalues of the correlation matrix $\mathbf{C}$ result in illconditioned precoding matrix and therefore noise enhancement when the typical ZF precoding is applied. As an advantage over ZF, for CI precoding, the ill-conditioning of the precoding matrix is mitigated. This is reflected especially in the low $\mathrm{Eb} / \mathrm{N} 0$ range in Fig. 4. For Eb/N0 values beyond $13 \mathrm{~dB}$, all the systems reach the maximum effective spectral efficiency $2.35 \mathrm{bit} / \mathrm{s} / \mathrm{Hz}$.

With further bandwidth saving to $20 \%$, which is equivalent to $\alpha=0.8$, the effective spectral efficiency comparisons are shown in Fig. 4. It is clearly seen that the maximum spectral efficiency is further increased to $2.5 \mathrm{bit} / \mathrm{s} / \mathrm{Hz}$. In addition, the gap between ZF precoding and CI precoding becomes wider. The ZF precoding assisted SEFDM is even worse than the theoretical QPSK in low Eb/N0 range between $1 \mathrm{~dB}$ and 7.5 $\mathrm{dB}$. This further explains the interference resistance of CI precoding. The CI precoding outperforms $\mathrm{ZF}$ precoding up to $\mathrm{Eb} / \mathrm{N} 0=19 \mathrm{~dB}$ and with no improvement beyond this point.

\section{EXPERIMENT VALIDATION}

The real-time experiment platform is presented in Fig. 6 and its device connections are illustrated in Fig. 7. This work is aimed at IoT communications. Therefore, instead of massive MIMO systems, only six transmission antennas are configured for the proof-of-concept purpose. In Fig. 7, six software

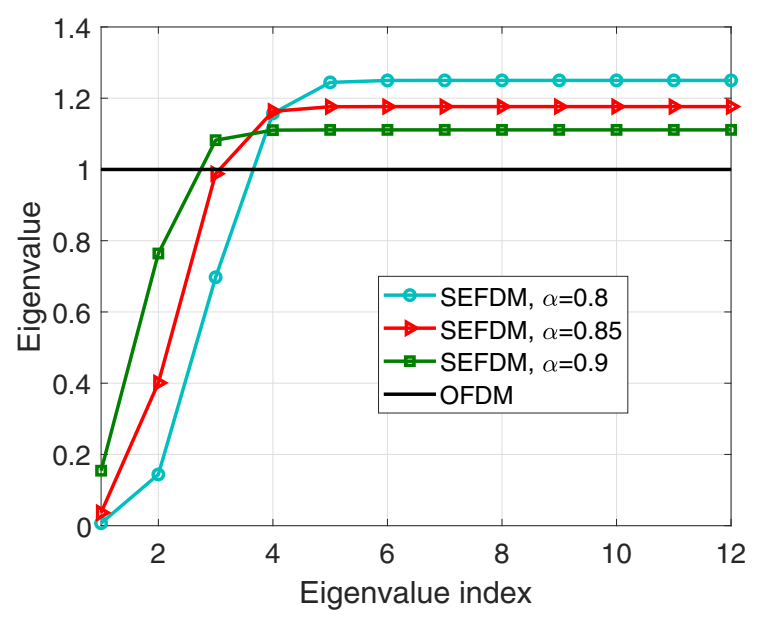

Fig. 5. Eigenvalues of the correlation matrix $\mathbf{C}$ versus eigenvalue index. $\mathrm{N}=12$ sub-carriers are tested.

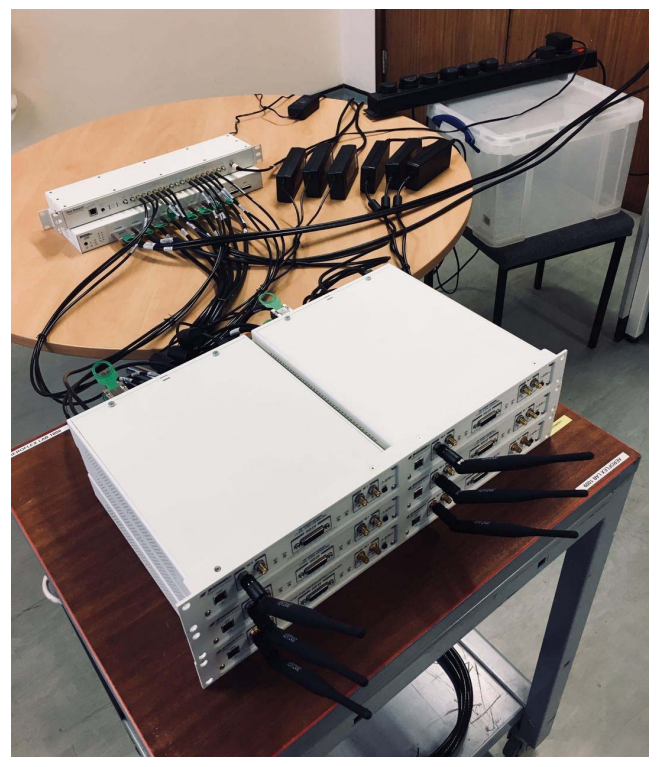

Fig. 6. USRP array setup for the MIMO-SEFDM precoding system.

defined radio USRP-RIO 2953R devices are connected to work as a base station. Each USRP has two separated and independent radio frequency (RF) chains and only one RF chain is activated in each USRP. In this case, six different data streams are generated in parallel and they have no correlations. The six USRPs are synchronized via SMA cables by a CDA2990 8-channel clock distribution OctoClock module, which is used to generate a $10 \mathrm{MHz}$ reference signal and a pulse per second (PPS) signal. In addition, raw data streams are originally generated from a work station and then sent to a cabled PCI-Express switch box CPS-8910 via an NI MXIExpress Gen $2 \times 8$ Cable, which can support data rate up to $3.2 \mathrm{~GB} / \mathrm{s}$. The switch box separates the data stream to six data streams and then delivered to six USRPs in parallel. The indoor over-the-air transmission link is realized via Omni- 


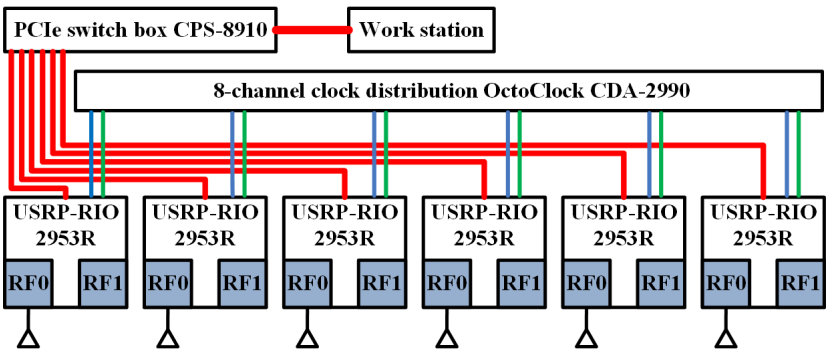

Fig. 7. System architecture and components connections. Red connections indicate NI MXI-Express Gen $2 \times 8$ cables. Blue connections indicate SMA cables delivering $10 \mathrm{MHz}$ reference signals. Green connections indicate SMA cables delivering PPS signals.

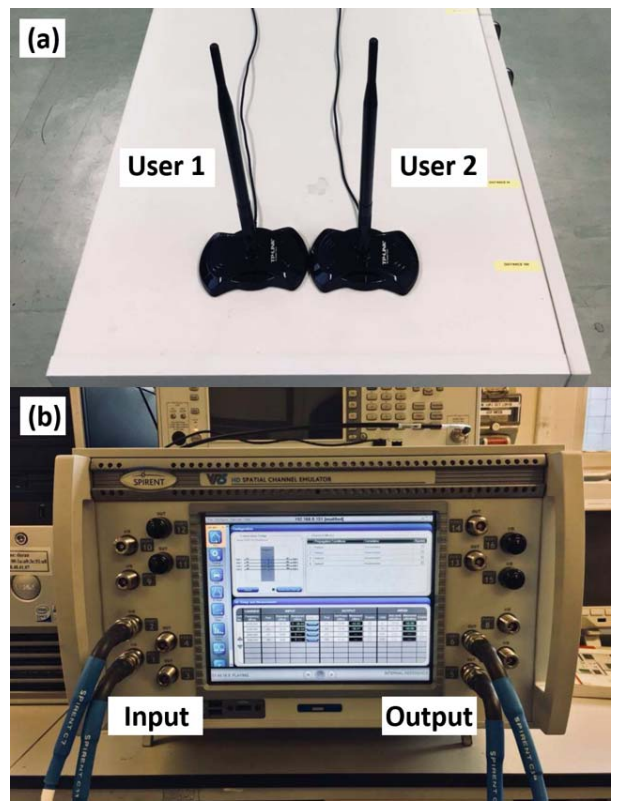

Fig. 8. (a) Receiver side user antennas. (b) Channel emulator VR5 for path loss and noise emulation.

directional antennas operating at $2.4 \mathrm{GHz}$.

At the receiver, two users are assumed and each user is equipped with one antenna as shown in Fig. 8(a). The distance between the base station and users is flexible up to 9 meters in the laboratory shown in Fig. 9. The experiment configurations for both the typical NB-IoT platform and the proposed NGIoT platform are presented in Table I.

Measured constellation results are illustrated in Fig. 10. The performance without precoding is shown in Fig. 10(a) where constellation points are scattered due to the self-created ICI. With ZF precoding, the constellation becomes better in Fig. 10(b) with four focusing points but some points around them. By using the CI precoding, the constellation is precoded and transformed into a special pattern in Fig. 10(c). It clearly shows that the CI precoding efficiently mitigates destructive interference near the hard decision edge via pushing signal points to the constructive region.

The experiment is limited by the indoor space of our labora-

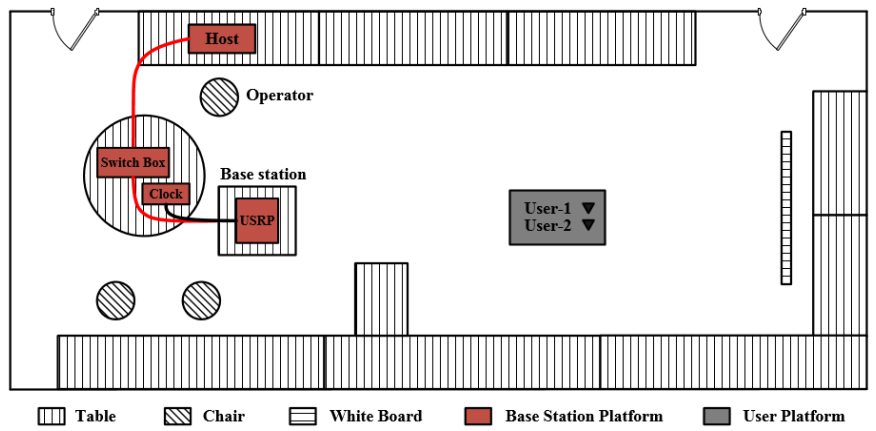

Fig. 9. Indoor floor plan and the location of USRP base station (has Tx antennas), user IoT devices (has Rx antennas), control host and experiment operator. The laboratory is around 9 meters long and 4 meters wide.

Table I: Experiment System Specifications

\begin{tabular}{lll}
\hline \hline Parameter & NB - IoT & NG - IoT \\
\hline No. of base station antennas & 6 & 6 \\
No. of users & 2 & 2 \\
RF center frequency $(\mathrm{GHz})$ & 2.4 & 2.4 \\
Sampling frequency $(\mathrm{MHz})$ & 1.92 & 1.92 \\
IFFT sample length & 128 & 128 \\
No. of guard band sub-carriers & 58 & 58 \\
No. of data sub-carriers & 12 & 12 \\
No. of cyclic prefix samples & 10 & 10 \\
Modulation scheme & $\mathrm{QPSK}$ & $\mathrm{QPSK}$ \\
Bandwidth Compression Ratio $(\alpha)$ & 1 & 0.85 \\
Sub-carrier bandwidth $(\mathrm{kHz})$ & 15 & 15 \\
Sub-carrier spacing $(\mathrm{kHz})$ & 15 & $15 \times \alpha$ \\
Maximum Spectral Efficiency $(\mathrm{bit} / \mathrm{s} / \mathrm{Hz})$ & 2 & $2 / \alpha$ \\
\hline \hline
\end{tabular}

tory, which is around 9 meters long and 4 meters wide. In order to obtain different SNR values, we vary noise power while maintaining the signal power using a commercialized channel emulator Spirent VR5 [16] as shown in Fig. 8(b). We assume noise power mainly comes from the receiver. Therefore, the VR5 is connected to two users at the receiver. Two input blue cables are separately connected to two user antennas. Received signals are transferred to the VR5 and contaminated by AWGN. Two output blue cables are connected to two USRPs, which are used for receiver side signal processing. The RF transmission signal power, coming from the base station, is $-35 \mathrm{dBm}$ and the receiver side signal is $-40 \mathrm{dBm}$. By tuning the noise power in the VR5 channel emulator, various SNR values are obtained. It should be noted that because of the resolution limitations of the VR5 channel emulator, the AWGN bandwidth is configured to $1.5625 \mathrm{MHz}$, which is wider than the signal bandwidth.

The effective spectral efficiency versus SNR is measured and illustrated in Fig. 11. It clearly demonstrates that in high SNR region, both CI precoding and ZF precoding systems reach the maximum spectral efficiency. However, in low SNR region, CI precoding aided SEFDM system achieves much better spectral efficiency. This practically verifies that CI precoding is more robust than ZF precoding in highly noisy channels. In addition, due to noise sensitivity, ZF precoding 


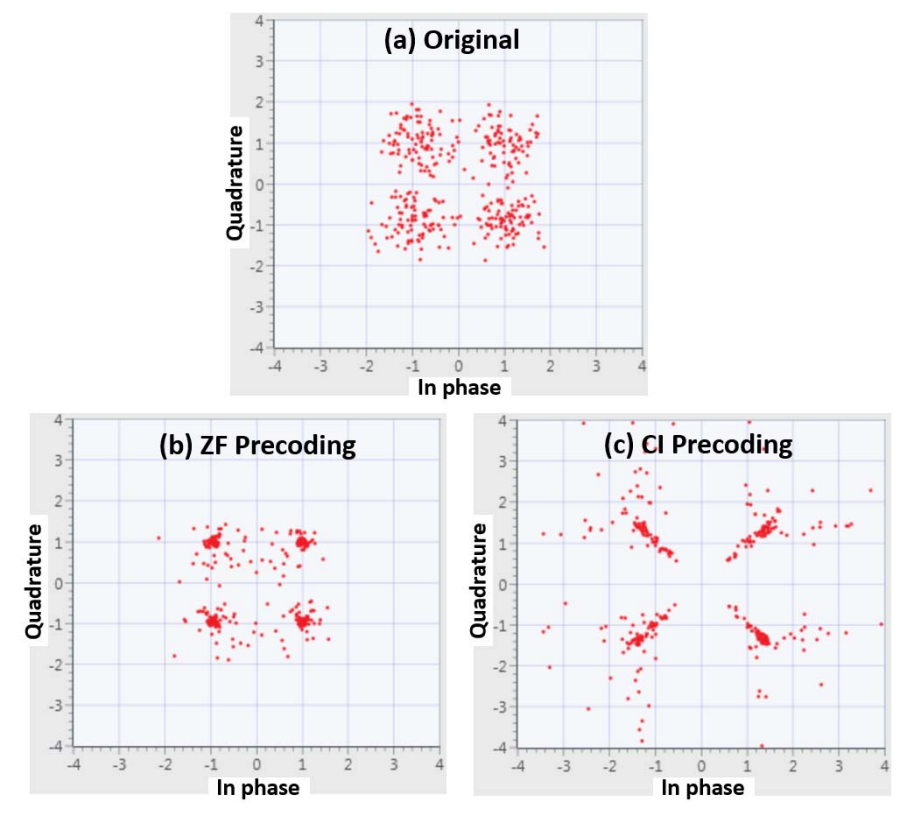

Fig. 10. Measured constellation patterns for non-precoding and precoding systems.

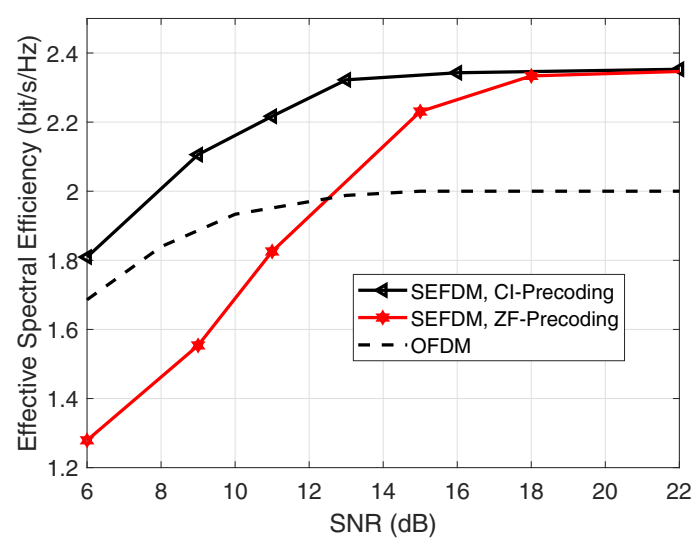

Fig. 11. Experimental spectral efficiency for the $18 \%$ spectral efficiency gain $(\alpha=0.85)$.

SEFDM performs even worse than OFDM at low SNR values.

\section{CONCLusions}

The non-orthogonal signal waveform, termed SEFDM, was tested in IoT communications bringing benefits such as spectral efficiency improvement. The main concern in IoT is power consumption due to the limited battery life in each IoT device. Therefore, precoding is used for downlink channels aiming to reduce signal processing pressure at receiver side IoT devices. ZF precoding is straightforward but it is sensitive to noise. A robust CI precoding method was applied for SEFDM and simulation results showed its superior performance than ZF precoding. In the case of small spectral efficiency gains, the benefit of CI precoding is not significant. With higher spectral efficiency gains considered, which indicates higher bandwidth compression ratios, CI precoding showed much better performance. It is concluded that CI precoding can reach the maximum spectral efficiency faster than ZF precoding when signals are distorted by the self-created ICI. In addition, practical experiment was operated on software defined radio USRPs to validate the simulation results. The CI precoding enables a distinct constructive constellation pattern, which improves the decision reliability leading to better spectral efficiency than ZF precoding. This guarantees reliable IoT communications using the non-orthogonal signal waveform.

\section{ACKNOWLEDGEMENT}

This work was funded by the Engineering and Physical Sciences Research Council (EPSRC) "Impact Acceleration Discovery to Use" award (Grant reference: EP/R511638/1) and Marie Sklodowska Curie fellowship. This work was also supported by National Instruments and Spirent.

\section{REFERENCES}

[1] B. Farhang-Boroujeny, "OFDM versus filter bank multicarrier," IEEE Signal Processing Magazine, vol. 28, no. 3, pp. 92-112, May 2011.

[2] N. Michailow, M. Matthe, I. Gaspar, A. Caldevilla, L. Mendes, A. Festag, and G. Fettweis, "Generalized frequency division multiplexing for 5th generation cellular networks," IEEE Transactions on Communications, vol. 62, no. 9, pp. 3045-3061, Sep. 2014.

[3] V. Vakilian, T. Wild, F. Schaich, S. Ten Brink, and J.-F. Frigon, "Universal-filtered multi-carrier technique for wireless systems beyond LTE," in IEEE Globecom Workshops, Dec. 2013, pp. 223-228.

[4] I. Darwazeh, H. Ghannam, and T. Xu, "The first 15 years of SEFDM: A brief survey," in 11th International Symposium on Communication Systems, Networks Digital Signal Processing (CSNDSP18), Jul. 2018, pp. $1-7$

[5] T. Xu, S. Mikroulis, J. E. Mitchell, and I. Darwazeh, "Bandwidth compressed waveform for $60-\mathrm{GHz}$ millimeter-wave radio over fiber experiment," Journal of Lightwave Technology, vol. 34, no. 14, pp. 3458-3465, Jul. 2016.

[6] Y. Wang, Y. Zhou, T. Gui, K. Zhong, X. Zhou, L. Wang, A. P. T. Lau, C. Lu, and N. Chi, "SEFDM based spectrum compressed VLC system using RLS time-domain channel estimation and ID-FSD hybrid decoder," in 42nd European Conference on Optical Communication (ECOC), Sep. 2016, pp. 1-3.

[7] J. Proakis and M. Salehi, Digital Communications. McGraw-Hill, 2008.

[8] S. Isam and I. Darwazeh, "Precoded spectrally efficient FDM system," in 21st IEEE International Symposium on Personal Indoor and Mobile Radio Communications (PIMRC), Sep. 2010, pp. 99-104.

[9] C. Masouros and G. Zheng, "Exploiting known interference as green signal power for downlink beamforming optimization," IEEE Transactions on Signal Processing, vol. 63, no. 14, pp. 3628-3640, Jul. 2015.

[10] A. Li and C. Masouros, "Interference exploitation precoding made practical: Optimal closed-form solutions for PSK modulations," IEEE Transactions on Wireless Communications., vol. 17, no. 11, pp. 76617676, Nov. 2018.

[11] National Instruments, "USRP-RIO 2953R, Software Defined Radio Reconfigurable Device," http://sine.ni.com/nips/cds/view/p/lang/en/nid/ 213005 .

[12] M. Chen, Y. Miao, Y. Hao, and K. Hwang, "Narrow band Internet of Things," IEEE Access, vol. 5, pp. 20 557-20 577, 2017.

[13] 3GPP TS 36.213 v.14.2.0, "LTE; evolved universal terrestrial radio access (E-UTRA); physical layer procedures," Rel. 14, Apr. 2017.

[14] P. N. Whatmough, M. R. Perrett, S. Isam, and I. Darwazeh, "VLSI architecture for a reconfigurable spectrally efficient FDM baseband transmitter," IEEE Transactions on Circuits and Systems I: Regular Papers, vol. 59, no. 5, pp. 1107-1118, May 2012.

[15] T. Xu, C. Masouros, and I. Darwazeh, "Waveform and space precoding for next generation downlink narrowband IoT," IEEE Internet of Things Journal, Jan. 2019

[16] Spirent Communications plc, "VR5 HD Spatial Channel Emulator," http://www.spirent.com/Products/VR5, Apr. 2018. 